\title{
LA RUMEUR \\ OU \\ LA SURVIVANCE DE L'INTEMPOREL DANS UNE SOCIÉTÉ D'INFORMATION
}

\section{Pascal Froissart ${ }^{1}$}

Les fleurs en celluloìd sont vraies à condition qu'on ne cherche pas à les faire passer pour de l'écaille.

Francis Picabia

La rumeur est une valeur à la hausse actuellement. Dans les médias en particulier: on se sert par exemple d'une "écriture rumorale"2 dans la titraille, comme "moyen d'accroche, même si le contenu de (l')article de presse lui «tord ensuite le cou»"3, voire comme tête de rubrique (par exemple, dans le Belgian Business Week). Un article sur quarante emploierait du reste le mot "rumeur" dans son contenu, dans les quotidiens généralistes $\left(2,5 \%\right.$ des articles $\left.{ }^{4}\right)$.

1 Candidat au doctorat conjoint en communication (Université du Québec à Montréal et Concordia University).

2 A. GRYSPEERDT et A. KLEIN, La galaxie des rumeurs, Bruxelles, Éd. E.V.O., 1995, (à parâitre), § 1.2.2.1.d.

3 Ibid, § 1.2.2.

4 Ibid., $\$ 1.1 .2 .75$ articles "rumorants" $\div 3012$ articles du corpus $=2,5 \%$.

Recherches en communication, $\mathrm{n}^{\circ} 3,(1995)$. 
Or il y a, dans la conception que l'on se fait du phénomène de la rumeur, un je ne sais quoi d'archaïque: le mode de diffusion de la rumeur rappelle trop une Gemeinschaft ${ }^{1}$ honnie et refoulée dans les sociétés autoproclamées "de l'information" ou de la communication (tautologie! diraient certains ${ }^{2}$ ). Le bouche-à-oreille, voyons, ce n'est pas convenable, à l'époque du satellite et des ordinateurs! Pourtant, les canaux informels sont constamment sollicités par la circulation des informations: $49 \%$ des Américains apprirent la nouvelle de l'assassinat de Kennedy par la voie du bouche-à-oreille... ${ }^{3}$

Le phénomène de rumeur est cependant distinct de celui de diffusion informelle des informations. Nous essaierons ici, en particulier, de cerner les trois aspects qui lient la rumeur dans son rapport au temps: sa prétention à l'actualité, son surgissement éphémère et cyclique à la fois, et en fin son mode d'apparition dont nous faisons l'hypothèse qu'il est plus conjoncturel que prévu.

\section{Rapide historique de la recherche}

Le sens du mot "rumeur" est plus récent qu' on ne le pense: il date approximativement de la dernière guerre mondiale, façonné par des chercheurs américains au service de l'Armée, à l'écoute de son moral ${ }^{4}$ et des propagandes ennemies. Indicateur fragile mais frappant de l'intérêt pour les rumeurs, un indicateur bibliométrique (cf. graphique) montre que les premiers articles datent du début du siècle ${ }^{5}$,

1 F. Toennies, cité par P. AtTallah, Théories de la communication. Histoire, contexte, pouvoir, Québec, Presses de l'Université du Québec, 1989. p. 8 à 17.

${ }^{2}$ L. SFez, Critique de la communication, Paris, Ed. du Seuil, 1990, p. 101.

3 P. B. Sheatsley et J. J. Feldman, "The Assassination of President Kennedy: A Preleminary Report on Public Reactions and Behavior", Public Opinion Quarterly, vol. 28, n² 2 (été), 1964, p. 189-215 (p. 193).

4 "Mais avec l'éruption de la Seconde guerre mondiale, l'attention [des chercheurs en sciences sociales] fut irrésistiblement attirée par ce sujet; il apparut bientôt évident qu'en temps de guerre, les rumeurs influençaient non seulement le moral des populations et sa confiance, mais qu'elles étaient en maints endroits des armes offensives de la propagande ennemie". R. H KNAPP, "A Psychology of Rumor", Public Opinion Quarterly, n ${ }^{\circ}$, 1944, p. 22-37.

5 Jung assimilait alors la rumeur à un "rêve" collectif (p. 43 \& 52). Cf. C. G. JuNG, "Contribution à la psychologie de la «rumeur publique»", p. 43-58, in Conflits de l'âme enfantine. La rumeur. L'influence du père, Paris, Aubier, 1935 (1910). 
mais que l'effort continu de recherche sur cet objet ne naît qu'au milieu du siècle ${ }^{1}$ et enfle de manière exponentielle jusqu'à nous ${ }^{2}$.

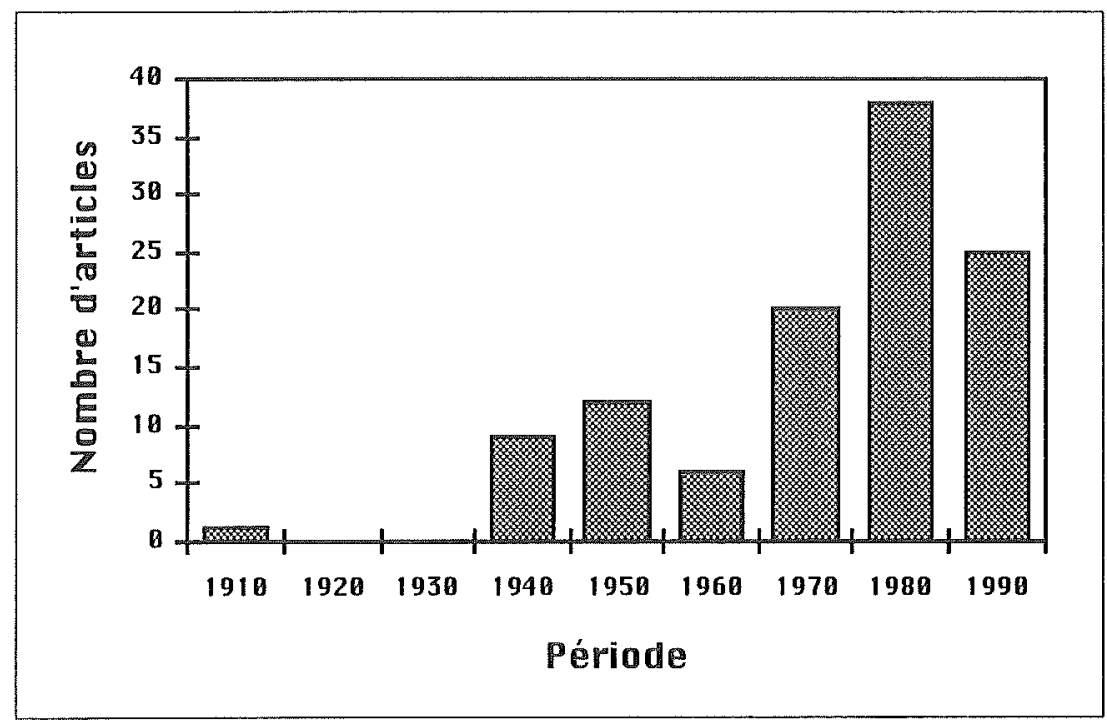

\section{Courbe bibliométrique de la rumeur}

Nombre d'articles publiés sur la rumeur, par décade. Base $\mathrm{n}=220$, bibliographie en langues française et anglaise établie dans P. FroISSART, La rumeur, un genre communicationnel à définir, (titre provisoire, en cours).

Sa présence dans le langage ne date pourtant pas de cette époque ${ }^{3}$. Expression empruntée au latin rumor, on en trouve les premières traces dans un édit de 1274 du Parlement de Paris où elle désigne tout autre chose: le "haro" que tout citoyen est obligé au nom

1 R. H. KnapP, op. cit. Allport, G. WILlaRd et L. J. Postman, The Psychology of Rumor, New York, Russel \& Russel, 1965 (1947).

2 Malgré certaines prédictions affirmant que "(...) les études relatives aux rumeurs soient progressivement moins nombreuses". P. MARC, De la bouche... à l'oreille. Psychologie sociale de la rumeur, Cousset, Delval, 1987, p. 239.

Les médias électroniques ne sont pas en reste: à preuve, le nombre d'émissions spéciales qui lui sont consacrées, dans la francophonie seulement (M6 [France], 9/1/94; SRC [Canada], 2/2/93; RSR1 [Suisse], 30/12/92; TF1 [France], 2/7/92, 16/7/92, 13/8/92; TSR [Suisse], 1988... pour les plus récentes).

3 Les données étymologiques proviennent de Fr. REUMAUX, "Rumor et opinio", Cahiers internationaux de sociologie, vol. $36, \mathrm{n}^{\circ} 86$, janvier-juin 1989, p. 129-132 et de B. PAillard, "L'écho de la rumeur", Communications, n 52, 1990, p. 125. 
de la loi de pousser s'il assiste à un crime, de manière à attirer l'attention de la maréchaussée! Son sens a ensuite lentement évolué au cours des siècles. Au XVII siècle, elle représente un bruit social incohérent, un chaos sonore porteur d'une signification diffuse. Au XVIII', elle désigne le bruit émanant d'une lutte ou d'une sédition, moins chaotique mais pas encore organisé, avant de se transformer le siècle suivant en l'expression de la dénonciation publique ou de la surprise devant un événement imprévu. À la fin du XIX ${ }^{\mathrm{e}}$ siècle seulement apparaît la différenciation des locuteurs; perdus dans une masse, dans le corps d'une foule anonyme, ceux-ci sont bientôt les acteurs de la rumeur. Naît alors l'idée de bouche à oreille et de bruit qui court: de ce fait la rumeur peut être injurieuse ou infamante, ce qu'elle ne pouvait signifier auparavant. Enfin, la rumeur du XXe siècle est le résultat d'une nouvelle évolution du concept, dans lequel on trouve un élément étrange mais significatif: la rumeur devient information, en plus de toutes ses caractéristiques antérieures.

Au début de son "existence", la rumeur est largement étudiée sous l'angle de la psychologie sociale et de l'adéquation entre le percept et le réel. Les études sur le témoignage de Binet et Claparède font tant école que la rumeur n'échappe à une conceptualisation en relation avec le témoignage: on établit alors une procédure dite "standard"1, tirée de la psychologie behaviorale ${ }^{2}$ et qui, même rebaptisée $\mathrm{MRS}^{3}$, n'est que la version plus ou moins scientifique du "téléphone arabe". Naturellement -mais c'était contenu dans l'énoncé du problème-, on constate l'absence totale de fidélité du témoignage tout au long d'une chaîne de sujets... Cette conception rumeur via information reste largement dominante dans les études récentes, qui résument parfois abruptement la rumeur à toute "information non vérifiée"4. Seules quelques brèches ont été faites par des études parcourues par des intérêts socio-psychanalytique ${ }^{5}$ ou ethnologique ${ }^{6}$.

AllPOR'T, G. WILlARD et L. J. PostMAN, op. cit., p. 65-74.

2 Fr. C. BARTletT, Remembering, Cambridge, Cambridge University Press, 1932.

3 MRS pour Méthode de répétition répétée, cf. O. KLINEBERG, "La mémoire", in Psychologie sociale, Paris, P.U.F., 1967, p. 246-256.

4 J.-N. KAPFERER, "Du Lsd dans les décalcomanies Mickey?", Actuel, nº 15, 1992, p. 36-38. Une rumeur serait "l'état provisoire, non vérifié, de l'information" (p. 36).

5 E. Morin, B. Pailla ard, É. Burguière, C. Capulier, C. Fischler, S. D E Lusignan, J. VéRONE, La rumeur d'Orléans, Paris, Éd. du Seuil, coll. 'L'histoire immédiate", 1969.

6 M. Roberge, La rumeur, Québec, Université Laval \& Célat, 1989. 
Que trouver à redire à cette conception dominante? Elle décrit bien une certaine réalité, le fait en particulier que certains récits informels circulant dans le corps social évoluent non seulement en énonciation mais aussi en signification - mais cela ne fait certes pas de la rumeur un objet autonome des sciences sociales. Tout au plus la face cachée de l'information journalistique.

Or nous prétendons nous saisir du concept de "rumeur dans le temps" pour tenter d'autonomiser le concept, contournant ainsi l'obstacle épistémologique ${ }^{1}$ que représente le sens commun, c'est-àdire ici l'adéquation à la réalité. Situer la rumeur dans le temps, c'est lui donner la chance de fonder une "unité de la représentation"2. Continuer à la considérer comme une variable dépendante de l'information, c'est la condamner à "n'être jamais autre chose qu'unité absolue".

Toute intuition contient en soi un divers, qui ne serait pas cependant représenté comme tel si l'esprit ne distinguait pas le temps dans la série des impressions successives, car en tant que contenue dans un moment toute représentation ne peut être jamais autre chose qu'unité absolue. Or, pour que de ce divers puisse sortir l'unité de la représentation, deux choses sont requises: le déroulement successif de la diversité, et la compréhension de ce déroulement, acte que je nomme la synthèse de l'appréhension ${ }^{3}$.

Donnons un exemple de notre démarche. Une rumeur très répandue en Amérique du Nord, répertoriée dans des livres à grande diffusion de type Vraies ou fausses? Les rumeurs ${ }^{4}$, raconte que des morceaux de souris décomposés (ou autres objets peu ragoûtants, préservatifs, aiguilles, etc.) ont été parfois retrouvés dans des canettes de Coca-Cola ou de boisson gazeuse. Eh bien, c'est véridique, c'est authentique (malheureusement...): 402 jugements de tribunal en font foi ${ }^{5}$ Q Que diraient ceux qui croient au schéma classique, où seule est valorisée l'adéquation de la représentation au réel? Ils affirmeraient: c'est arrivé une fois (au moins), c'est vérifié, c'est officiel; ils

G. BACHELARD, La formation de l'esprit scientifique, Paris, P.U.F., 1970.

2 E. KANT, Critique de la raison pure, Paris, Gallimard, coll. "Bibliothèque de la Pléiade", 1980, (1781, 1ère édition), p. 1406.

3 Ibid.

4 H. Morgan, K. Tucker et M. Voline, Vraies ou fausses? Les rumeurs, Paris, First, 1988, p. 72.

5 Fr. ReumauX, "L'actualité de la rumeur", Société, n 31, 1991, p. 15-20. 
concluraient, et le sens commun avec eux, que le témoignage rapporté, c'est-à-dire la représentation, est adéquate. La rumeur serait aussitôt "élucidée" et transmuée aussitôt en information "vraie".

Pourtant, la réalité est plus complexe et demande à ce qu'on "plonge" le phénomène linguistique dans l'espace temporel et social: le fait que le contenu de cette rumeur soit vérifié n'explique en rien pourquoi celle-ci circule de manière endémique depuis plus d'un demi-siècle. Ni qu'elle circule au présent, pour décrire une réalité actuelle, un acte ponctuel, alors que les faits rapportés remontent parfois jusqu'en 1914 (date du premier procès ${ }^{1}$ )! Tous les locuteurs de la rumeur la résument dans une phrase du genre «J'ai un $\mathrm{ADUA}^{2}$ qui a un jour trouvé un morceau de verre dans son Fanta». Il n'y est question que d'une performance individuelle, performée par une personne seule, à l'aide d'un performatif topique. Mais non d'une réalité sociale, partagée par plusieurs centaines d'Américains... Le texte de la rumeur fait donc abstraction de la temporalité du phénomène, que notre propos voudrait cependant rétablir. Remettre un phénomène intemporel dans sa réalité temporelle est notre propos.

\section{Machine à transformer le passé en présent atemporel: la prétention à l'actualité de la rumeur}

Plaidoyer pour la prise en compte du phénomène social en parallèle à l'étude du texte, cette position théorique ne peut pas faire abstraction des phénomènes sémantiques à l'œuvre dans le message, sitôt qu'on l'éloigne de son rapport pur à la véracité. Ainsi que pour les matériaux folkloriques ${ }^{3}$, des processus d'actualisation sont à

1 G. A. Fine, "Cokelore and Coke Law: Urban Belief Tales and the Problem of Multiple Origins", Journal of American Folklore, vol. 92, n³66, octobredécembre 1979 , p. 477-482.

2 L'acronyme ADUA désigne en français l'ami d'un ami, sur le mode de constitution du terme anglais FOAF, pour friend of a friend. Dans les deux cas, il s'agit de celui à qui est supposément arrivée l'aventure. Acronyme inventé et popularisé par R. DALE, The Tumour in the Whale. A Collection of Modern Myths, London, Duckworth, 1978. Il donne même lieu à la publication d'un périodique, FOAFtale News...

3 Van Gennep énonce pour cela une loi «de la temporation et de la détemporation» pour expliquer la mise hors du temps des légendes. A. VAN GENNEP, La formation des légendes, Paris, Flammarion, 1929, (1910), p. 280. 
l'œuvre, "l'actualité échappant à la discontinuité de l'éphémère parce qu'articulée sur (...) les archétypes et la mémoire collective"1, ramenant toujours la rumeur à un présent atemporel, car hors du temps, dans un temps irréel parce que toujours présent. "La rumeur est hors-temps. La rumeur prend la forme et la couleur de notre temps mais elle parle d'un autre temps. Celui que l'on peut qualifier de mythique parce qu'il est «trans-historique» ${ }^{2}$. La rumeur, dans son "combat" contre le temps humain, n'hésite même plus à proposer des récits anciens sous le jour d'une histoire moderne. Dans un cas même, on a pu retrouver un conte traditionnel passé dans l'univers rumoral: l'histoire de ce fils prodigue qui est revenu anonymement chez lui, après une longue absence; eh bien, il s'est fait dévaliser et assassiner par ses parents aubergistes en mal d'argent ${ }^{3}$.

L'actualité de la rumeur est feinte par l'actualisation; ou plutôt, l'actualité est un construit élaboré au cours de la transmission orale:

C'est la rumeur elle-même qui crée l'actualité: ce qui n'existe pas dans le discours du groupe n'existe pas en effet pour le groupe et l'actualité se définit comme ce qui est "actualisé" par la parole sociale 4 .

En définitive, l'actualité est la forme rituelle d'énonciation de la rumeur, texte parmi d'autres de littérature orale. C'est pourquoi même les rumeurs de prédictions sont racontées au présent...

Une amie d'un de mes amis, qui est lui-même un réfugié de l'Irak, était dans le tramway et quand le conducteur se présenta pour recueillir le prix des billets, la dame en face d'elle dit: Je vais payer pour vous car vous n'avez pas d'argent dans votre porte-monnaie. - Mais j'en ai, répliqua-t-elle. Cependant en ouvrant son porte-monnaie, elle découvrit que c'était vrai. - Mais pouvez-vous aussi lire dans l'avenir, ajouta-t-elle, quand la guerre sera finie? - Je suis une voyante, dit la femme, la guerre sera terminée le 15 mars.

1 Fr. ReumauX, op. cit., p. 19-20.

2 A. GRYSPEERDT et A. KLEIN, op, cit., § 1.3.

3 Fr. Reumaux, "Un rite oral urbain: la rumeur", Cahiers de littérature orale, $\mathrm{n}^{\circ} 24$, 1988, p. 62-63.

4 M.-L. RouQueTte, Les rumeurs, Paris, P.U.F., coll. «Sup», 1975, p. 19. 
Cet exemple, reprenant la fameuse "rumeur de prédiction" qui fit le tour de 1'Europe lors de la Seconde guerre mondiale", montre comment la rumeur met en avant, au passé, une actualité brûlante: le temps du méta-récit ("une amie était") donne à la prédiction l'enveloppe narrative de l'événement, et lui confère cet étrange aspect atemporel en mélangeant allègrement futur, passé et présent... La rumeur, dans ce maelström temporel, semble "se diffracte(r) en plusieurs degrés et niveaux de sens":

le temps du récit proprement dit, qui est le temps de la fiction, un temps intérieur puisant dans la mémoire et l'imaginaire social les dits dont elle est faite, le temps propre de la transmission qui renvoie à la réalité immédiate du temps structurel et conjoncturel.

Ces trois temps diffractés, pour reprendre l'image reumaldienne, font d'elle un labyrinthe dans lequel les journalistes, et autres passionnés de l'information, se perdent à l'envi... avant de s'en apercevoir (parfois!) après coup. «Il s'est fait avoir!», s'exclamait ainsi un chroniqueur montréalais ${ }^{2}$ en guise d'erratum, en parlant de lui-même, trois jours après avoir publié sans le savoir le récit de l'Elevator Incident:

L'histoire raconte qu'un homme de peau noire se trouve dans un ascenseur avec son chien, en compagnie de trois femmes de peau blanche. L'homme intime un ordre au chien - «Assis!»-, mal interprété par les jeunes femmes, qui s'assoient par terre ${ }^{3}$.

Le journaliste avait relaté l'histoire après que sa "source" la lui a racontée comme entièrement originale. Il n'y avait pas vu malice, sa source non plus... et tout le monde avait été attrapé par l'atemporalité de cette histoire racontée de manière répétée et quasi similaire aux États-Unis depuis les années soixante! Machine à transformer le passé en présent atemporel, la rumeur se mue et transmue en un événement

1 M. BonaPARTe, Mythes de guerre, (Londres) Paris, (Imago) P.U.F., (1946) 1950, p. 51.

2 J. Todd, "He was had", The Gazette, 10 février 1992, p. a3.

3 L'homme se met à rire à gorge déployée; plus tard, les jeunes femmes trouveront un mot de cette personne, signé d'un nom célèbre. J. H. BRuNVAND, The Mexican Pet - More «New» Urban Legends and Some Old Favorites, New York \& Londres, W.-W. Norton \& $\mathrm{C}^{\circ}, 1986$, p. 45. 
sans date, perpétuellement répété dans la pensée sociale, mâché et remâché comme un grand "chewing-gum intellectuel"1.

Cependant, l'actualité perpétuelle de la rumeur, analysée per se, ne saurait faire oublier la temporalité pragmatique de la rumeur étudiée en situation. Ainsi peut-on saisir le phénomène social dans une globalité sociale, qui permet de "relativiser le concept d'actualité et son corrélatif, l'immédiateté, volontiers reconnu à toute rumeur, l'immédiateté ne caractérisant que le stade d'éclosion"2.

\section{L'apparition éphémère et cyclique du message à prétention d'actualité}

La rumeur, certes, crée sa propre actualité, grâce à un processus d'actualisation similaire à celui à l'cuvre dans les récits traditionnels. Mais elle n'apparaît pas au hasard du temps: de longtemps, les "rumorophiles" ont constaté que ces récits à prétention d'actualité reviennent, naissent et meurent, et puis renaissent... Incompatibilité? Non, élément constitutif du phénomène: la rumeur est précisément caractérisée par cet aspect apparemment contradictoire, l'énonciation répétée d'un message prétendant à l'actualité. C'est une des «traits invariants de la rumeur», bien que le mode d'apparition de la rumeur soit loin d'être élucidé. Est-ce un phénomène cyclique, ou simplement la renaissance aléatoire d'une légende moderne ${ }^{3}$ Les réponses diffèrent, selon la conception personnelle que les chercheurs ont de l'origine de la rumeur (ou du cycle): ceux qui voient dans la rumeur un récit similaire aux légendes lui donnent volontiers une personnalité cyclique, tandis que les autres la classent parmi les symptômes sociaux de crise et de tensions.

En tout état de cause, la rumeur est le lieu d'une succession de modèles d'apparition dans le temps: une succession de silences et d'explosion. Les poètes le chantaient déjà pour la renommée, “aux pieds rapides, aux ailes promptes, monstre horrible, énorme, qui a

1 Lumley, 1925, cité par R. L. Rosnow et G. A. Fine, Rumor and Gossip. The Social Psychology of Hearsay, New York, Elsevier, 1976, p. 84.

2 Fr. ReumauX, "L'actualité de la rumeur", op. cit., p. 15.

${ }^{3}$ Le concept d'origine anglo-saxonne de "légende contemporaine" vient parfois se substituer à cet endroit de la caractérisation de la rumeur: comme elle, ce sont des récits à prétention d'actualité, mais dont le mode d'apparition n'est pas explicité. 
autant d'yeux perçants que de plumes, ô prodige, autant de langues et de bouches sonores et d'oreilles dressées"1 ou pour la calomnie:

D'abord un bruit léger, rasant le sol comme hirondelle avant l'orage, pianissimo murmure et file, et sème en courant le trait empoisonné. Telle bouche le recueille, et piano, piano, vous le glisse en l'oreille adroitement. Le mal est fait; il germe, il rampe, il chemine, et rinforzando de bouche en bouche il va le diable; puis tout à coup, ne sais comment, vous voyez calomnie se dresser, siffler, s'enfler, grandir à vue d'œil. Elle s'élance, étend son vol, tourbillonne, enveloppe, arrache, entraîne, éclate et tonne, et devient, grâce au ciel, un cri général, un crescendo public, un chorus universel de haine et de proscription. Qui diable y résisterait? ${ }^{2}$

Le mode d'apparition est l'une des caractéristiques de la rumeur la plus partagée par les chercheurs en sciences sociales - à part la distinction subtilement amenée des signes externes de la circulation d'une rumeur. Le problème, même s'il ne pose pas réellement problème, mérite qu'on s'y arrête: comment juger de la diffusion d'une rumeur. Certains proposent qu'on ne retienne que

la partie manifeste, audible, de la rumeur, celle où, parvenue à mobiliser les attentions autour de ses dires, elle recentre un groupe ou une communauté autour d'un nouvel objet ${ }^{3}$.

D'autres incluent dans le phénomène de rumeur des phases supputées de latence, après lesquelles la rumeur "s'exprime", comme le ferait un gène récessif... Morin et al. décrivent le "cycle complet à Orléans" de la rumeur.

Proliférant sur un germe dont on peut déterminer la double origine mythologique et la même source fantasmatique, elle incube (10-20 mai), entre en virulence et en extension rapide (20-27 mai), se déchaîne en une prodigieuse métastase (29-31 mai), se disloque sous la contre-attaque (2-10 juin), régresse dans le fantasme et les mini-rumeurs, s'enfonce dans l'amnésie, laisse des résidus et des germes ${ }^{4}$.

1 VIRGILE, L'Énéide, IV, v. 173-188, Paris, Les Belles lettres, 1970, p. 123.

2 BeAumarchais, Le barbier de Séville, acte II, scène VIII.

3 Bysow cité par Fr. REumauX, "Traits invariants de la rumeur", Communications, $n^{\circ} 52,1990$, p. 142.

4 E. MORIN et al., op. cit., p. 36-37. 
Cycle de vie que Reumaux reprend à sa façon, en utilisant la métaphore du papillon (certes plus poétique que les cancers...) et en décomposant en trois "stade socio-temporels" : de larve à imago, en passant par l'étape de nymphe, la rumeur est prête à prendre son envol.

D'autres enfin distinguent plusieurs types de rumeurs en fonction précisément des modes d'apparition de la rumeur: ainsi Bysow ${ }^{2}$ proposait-il de classer les rumeurs suivant leurs manifestations visibles. Il distinguait trois types de rumeur, celle qui rampe (jusqu'à ce que tout le monde la connaisse), celle qui fait feu de paille (et qui a généralement un sujet touchant à un péril immédiat), et enfin la rumeur "sous-marine" (de nature essentiellement cyclique).

La phase de résurgence de la rumeur est naturellement la plus documentée: pour des raisons autant matérielles (il est plus aisé de garder trace de manifestations publiques, de plaintes policières, ou de tracts, que de mots vites envolés, fussent-ils "à la base" des premiers...) qu'émotives (la puissance des mots, la folie du socius en crise, tout cela fascine... et les artistes ne sont pas en reste à décrire une réalité sociale qui parfois s'échappe à elle-même). On répertorie donc sans problème les résurgences de la rumeur dite «d'Orléans»: en 1966 à Dinan, Laval, et Rouen; en 1968 au Mans; en 1969 à Orléans, Poitiers, et Châtellerault; en 1970 à Amiens; en 1974 à Châlon-surSaone; et même en 1985 à Dijon et La Roche-sur-Yon ${ }^{3}$. De la même manière, on repère aisément, dans les journaux en particulier, les manifestations de la rumeur dite «des auto-stoppeurs fantômes»: du XVII siècle $^{4}$ jusqu'en septembre 1992 (au moins) où une femme en chandail rayé a défrayé la chronique des journaux de la ville québécoise de Chicoutimi (photos de la rédaction à l'appui) ${ }^{5}$, de la Wallonie

1 Fr. Reumaux, Toute la ville en parle .Esquisse d'une théorie des rumeurs, Paris, L'Harmattan, 1994, p. 8-9.

2 L. A. BYSOW, (trad.: N. VON WIESE), "Gerüchte", Kölner Vierteljahrschefte für Soziologie, 1928, nº 7, p. 421.

3 J.-N. KAPFERER, Rumeurs.. Le plus vieux média du monde, Paris, Éd. du Seuil, 1987, p. 143.

Pour rester sur le seul territoire français. Car on la repère à Montréal en $1992 \ldots$ et en Corée en 1987. Cf. P. FroISSART, Quelques concepts qui servent de base à l'étude de la rumeur - Réponse à la première question de l'examen de synthèse, Montréal, Université du Québec à Montréal, 1992.

4 Fr. Dumbrchat, "Les auto-stoppeurs fantômes", Communications, Paris, $n^{\circ} 52$, 1990, p. 249-282.

5 P.-E. Thériault, et N. Boivin, "Fantôme du parc des Laurentides — La réalité dépasse parfois la fiction", Le Progrès-Dimanche, 6 septembre 1992, p. A8. 
à l'Ohio ${ }^{1} \ldots$ Elle surgit en tous temps, en tous lieux... sans prévenir, et apparemment sans motif. On peut essayer d'établir des liens entre les dates et d'en déduire des cycles, mais on se heurte toujours au même problème d'appréhension du phénomène: avec de tels outils, on en tirerait tout au plus le cycle des manifestations de la rumeur (émeutes, publications, etc.) mais non la diffusion de la rumeur elle-même. On risquerait d'être plus positiviste que Comte lui-même, et de vouloir enfermer à l'aide d'une seule variable dérivée l'ensemble du phénomène.

Pour conclure partiellement sur la discontinuité du phénomène, ce caractère éphémère et récurrent influence évidemment la créance des auditeurs ${ }^{2}$, mais de trois manières différentes: elle peut lui faire l'effet de renforcement (je le crois car je l'ai déjà entendu; je le crois car tout le monde le croit); ou au contraire un effet de défiance (je ne le crois pas car je l'ai déjà entendu); enfin, comme nous en ce moment, un intérêt nosologique par la mise en évidence d'un thème, d'une rhétorique, ou d'un mode de narration déjà rencontré dans l'un de ces nombreux recueils de rumeurs ou de légendes contemporaines... ${ }^{3}$

\section{L'apparition conjoncturelle de la rumeur: le tremplin de la crise}

Vue seulement comme un phénomène éphémère et cyclique, la rumeur ne se distingue pas réellement des autres phénomènes qui l'accompagnent sur la voie de l'oralité et de la narration rituelle (ainsi les contes aussi sont éphémères et cycliques, bien que rarement des rumeurs). Nous proposons d'invoquer une hypothèse sociologique pour faire de la rumeur un fait social en lui-même - et non seulement un donné linguistique: la rumeur reste latente tant que des faits sociologiques ne viennent la déloger de son calme sous-jacent. Par le

1 J. H. BRUNVAND, The Vanishing Hitchhiker. American Urban Legends and Their Meaning, New York \& Londres, W.-W. Norton \& $C^{\circ}, 1981$.

2 On désignera par le terme d'“auditeurs" les personnes qui ont été interrogées, bien que, en plus d'être auditeurs, les personnes qui écoutent une rumeur deviennent acteurs quand elles la diffusent.

3 Par exemple, Morgan, Hal \& al., op. cit.; V. CAMPion-Vincent, et J.-Br. RENARD, Légendes urbaines - Rumeurs d'aujourd'hui, Paris, Payot, 1992; ou J. H. BRUnVAND, op. cit.; cf. également la grosse base de données sur Internet, stockée au Mit (alt.folklore.urban) sous la direction de Brunvand lui-même. 
court exposé d'une rumeur que nous avons étudié par chance ${ }^{1}$ in situ, nous voudrions montrer que la rumeur est volontiers conjoncturelle.

En avril 1992, une rumeur ${ }^{2}$ secoua la capitale de la Guinée et devint le centre de toutes les conversations. Son contenu était pourtant, comme toujours, anecdotique - bien qu'extrêmement impliquant et "actuel": «Un ressortissant espagnol aurait livré sa compagne, ou ses compagnes guinéennes, à... son chien», résumait la presse indépendante ${ }^{3}$, omettant, en les sous-entendant, de nombreux détails: "Le Blanc était là, au moment de... entre la fille et le chien. Et il prenait des photos"4; "Le Blanc disposait de caméra cachée dans la chambre. Il filmait la scène...". Il en organisait aussi le trafic: "Il prenait des photos qu'il revendait ensuite chez eux." Le gardien du riche expatrié va raconter dans le quartier ce qui se passe chez le Blanc; il va briser le secret d'alcôve: "Il filmait la scène et il a été surpris par son gardien. Celui-ci s'est rendu chez les vieux du quartier pour se plaindre de l'attitude de la fille."; "C'est le gardien qui révèle tout. Il est allé à la mosquée. Après la prière, il s'est adressé à quelques sages, des vieux, et leur a tout révélé "en tant que bon musulman": un Blanc, des filles, etc.".

Cette rumeur aurait pu paraître anodine pour un observateur étranger -sordide, tout au plus. Pour la population de Conakry, ce fut un bouleversement. On apprit bientôt que la villa du ressortissant espagnol avait été rasée par une population en furie ${ }^{5}$; des tracts pornographiques se mirent à circuler de main en main ${ }^{6}$; des explosions émeutières secouèrent la ville. Les médias d'État s'en

I J'assurais pour le compte du Ministère français de la coopération un enseignement en journalisme en Guinée-Conakry.

2 Cf. pour plus de détails "La rumeur du chien", in P. FroISSART, La rumeur, un genre communicationnel à définir (titre provisoire), thèse de doctorat en communications (en cours), Montréal, Université du Québec à Montréal, 1995.

3 A. Diomandé, "Violence à Ratoma", Lynx, n 9, 20 avril 1992, p. 4.

4 Les citations en italique et entre guillemets sont issues d'une enquête ethnosociologique, auprès d'un échantillon aléatoire de 80 résidents de Conakry, effectuée dans les langues nationales (soussou, mandingue, pular ou langues forestières) ou en français par des enquêteurs guinéens sous notre direction.

5 Conduit par l'un de nos enquêteurs, nous avons pu en voir les restes: le toit était effondré, il ne restait plus que les murs - sur l'un desquels nous pûmes apercevoir le dessin enfantin d'un énorme chien.

6 A prix d'or, indice s'il en faut de la très forte implication des Conakrykas. Vendues de main en main le long des trottoirs, le prix de ces photocopies de photocopies variait entre 500 et $2000 \mathrm{FG}$ (à titre de comparaison, un repas normal pris dans un "maquis", restaurant populaire, vaut $300 \mathrm{FG}$ ). 
saisirent, citant noms des protagonistes et lieux, les téléscripteurs de l'AFP se mirent à crépiter...

Des Guinéens ont manifesté le 20 avril dans le quartier de Madina, le plus peuplé de Conakry, brutalisant les jeunes femmes portant un pantalon ou une minijupe. À l'origine de ces violences, l'arrestation, la veille, d'un ressortissant espagnol qui aurait tourné secrètement des films pornographiques à son domicile. Les habitants du quartier ont failli le lyncher et s'en sont pris ensuite aux automobilistes européens de passage dans le quartier, qu'ils ont molestés. Le ministre de l'Intérieur a présenté ses excuses aux Européens victimes, ou menacés. L'enquête de police aurait révélé que l'affaire n'était en réalité qu'une "pure machination dénuée de tout fondement"l.

Il fallut que le Ministre de l'intérieur puis le Chef de l'État luimême vinssent à la télévision lancer des appels au calme pour qu'enfin l'agitation cessât (on releva au moins un mort ${ }^{2}$, des dizaines de blessés, et il y eut 45 arrestations ${ }^{3}$ ). Mais les langues continuèrent de fredonner le refrain de la rumeur...

Les explications de sens commun ne se suffisent pas à ellemême. Penserait-on que la bestialité soit taboue? Certes mais des légendes guinéennes la mettent en scène sans causer d'autres dommages qu'éclats de rire et explosion d'hilarité ${ }^{4}$. De plus les pratiques zoophiliques sont universelles, tout autant que les interdits traditionnels et légaux qui les régissent ${ }^{5}$. Enfin, il existe une bestialité rituelle dans de nombreuses régions d'Afrique, en particulier dans le Golfe de Guinée où les Ijaw du Nigeria pratiquent "un coït rituel avec les antilopes et les brebis, soit pour préparer le succès de la chasse, soit au cours des cérémonies d'initiation des garçons" 6 .

1 Dépêche reprise dans Marchés tropicaux et méditerranéens, 24 avril 1992, p. 1047.

2 I. Sow, "Une journée chaude: un mort, une blessée grave et des dégâts matériels", Horoya, vol. $31, \mathrm{n}^{\circ} 3646,15-17$ avril 1992, p. 3.

3 M. Koulibaly, "Pour le retour de la quiétude", Horoya, vol. $31, \mathrm{n}^{\circ} 3646,15-17$ avril 1992, p. 5.

4 Un conte populaire peulh, par exemple, met en scène les organes génitaux d'un âne et passe allègrement sur les tabous de la bestialité, du fétichisme, du voyeurisme et même de la sodomie (V. Fr. ÉQUILBECQ, Contes populaires d'Afrique occidentale, Paris, A. Maisonneuve, 1972 (rééd.), p. 326-327).

5 R. VILLENEUVE, Le musée de la bestialité, Paris, Veyrier, 1973, p. 9-13.

6 B. DE RACHEWILTZ, Éros noir - Mours sexuelles de l'Afrique de la préhistoire à nos jours, Paris, (La Jeune Parque) Le Terrain vague, (1965) 1993, p. 283. 
L'écart à la moralité est-il un fait suffisant à mobiliser tous les Guinéens? Certains l'ont affirmé ("Cela blessait la dignité des Guinéens", confiait un journaliste), mais une rumeur ne peut se diffuser que si elle arrive à impliquer tous les auditeurs qu'elle touche; or il n'existe pas d'indice pour penser, sauf peut-être dans un rêve intégriste, qu'une population entière soit mobilisée par le même motif moral.

Le soudain embrasement de la citél est d'autant moins compréhensible que la rumeur avait déjà circulé, ainsi que le veut la loi de récurrence que nous avons précédemment exposée, quelques années auparavant, dans une région voisine de la capitale. Un tiers des auditeurs interrogés l'avaient déjà entendue en 1977 ou 1979 en Moyenne Guinée. Mieux que cela, un haut fonctionnaire français en poste à Yaoundé (Cameroun) nous a certifié qu'elle y avait eu cours en 1982 à quelque $2800 \mathrm{~km}$ de là! Comment se fait-il que, cette foisci, non seulement elle ait été crue par tous, mais en plus elle ait causé tant de dégâts?

Nous voudrions lier cette soudaine déflagration sociale avec les tensions qui étaient alors présentes en Guinée. Car, sans toutefois proposer la vision déterministe d'une rumeur engendrée par la crise ${ }^{2}$, nous voudrions y voir un lien de concomitance. Or ce lien entre le surgissement des signes manifestes de la rumeur avec la "situation de crise" avait été vu par Morin et al. à l'orée de la rumeur d'Orléans, apparue en pleine campagne électorale. Ainsi rapportent-ils que 1'un des commerçants visés par la rumeur vint au commissariat pour porter plainte et que le commissaire le conseilla en ces termes: "Attendez quarante-huit heures, et après allez-y". Il s'agissait "de ne pas perturber les deux ultimes journées pré-électorales"3.

Ici, il convient de se demander s'il y a eu quelque relation entre la rumeur et la crise présidentielle de mai 1969. (...) Celle-ci a correspondu à un phase d'incertitude, d'inquiétude qui, puissamment refoulée hors de la conscience politique, aurait dérivé vers les bas niveaux inconscients. (...) Il n'est

1 Conakry compte 1,5 millions d'habitants, soit à peu près autant que Bruxelles, Montréal ou Bordeaux...

2 Pour la notion de crise dans laquelle s'insère la rumeur, cf. M. RouquetTe, $L a$ pensée sociale et les phénomènes de rumeur, Université d'Aix-Marseille I, thèse de doctorat d'État s. dir. Claude Flament, 1980, p. 162-166.

3 E. Morin et al., op. cit., p. 95. 
pas exclu que ce fut l'appoint décisif pour le déclenchement de la métastase ${ }^{1}$.

Dans une étude similaire (une rumeur de sorcellerie au Togo), Claude Rivière ${ }^{2}$ avait noté également la corrélation entre le surgissement de la rumeur et des facteurs externes comme le prix des céréales (le prix du mais, aliment de base au Togo, avait connu de grosses variations dans les mois qui avaient précédé le surgissement de la rumeur).

La rumeur du chien n'échappait pas à la règle: au même moment où surgissait la crise rumorale, de très nombreux facteurs de tension sociale étaient en place. La vie politique était en effet bouillonnante. Le pays venait de se doter de lois organiques qui autorisaient le multipartisme (le bipartisme avait été légalisé l'année précédente seulement). Du coup, il ne se passait plus un mois depuis le début de l'année sans que se créât un nouveau parti. Dans la seule semaine du 16 mars (soit trois semaines avant le déclenchement de la rumeur), ce sont six nouveaux partis qui ont déposé leurs statuts au Ministère de l'intérieur! Les réianions politiques se succédaient les unes aux autres, la ville bruissait de discussions politiques et de meetings. Enfin, le gouvernement avait subi un septième remaniement au mois de février. Dans un pays habitué au silence des milices jusqu'en 1984 (année de la mort de Sékou Touré et du début de l'ouverture du pays vers l'extérieur), on imagine la répercussion phénoménale de ces événements. De plus, la Guinée sortait de la période du Ramadan (du 5 mars au 4 avril), dont le jeûne forcé durant les heures du jour a de quoi exacerber les tensions les plus bénignes. Influente aussi était la hausse des prix que la capitale connaît quasi systématiquement à cette époque.

Le Ramadan saint est, en Guinée, un mois où les spéculateurs font la loi sur les marchés. Profitant de la forte demande en produits de première nécessité, les marchands multiplient les prix à qui mieux mieux ${ }^{3}$.

1 Ibid., p. 29-30.

2 Cl. Rivière, "Rumeur de métamorphose", Ethmopsychologie, vol. 33, n 1, janviermars 1973, p. 64.

3 J. DRAMOU, "Ramadan et flambée des prix", Horoya, vol. 31, n 3640, 16-19 mars 1992, p. 8. 
Les augmentations étaient substantielles (25\% sur la plupart des produits: oignons, gombo, huile d'arachide...), mais quelquefois aussi gigantesques ( $200 \%$ sur la salade). Le riz même, aliment central de l'alimentation ouest-africaine, subit des variations à la hausse (le sac de $50 \mathrm{~kg}$ passe facilement de 15000 à $17000 \mathrm{FG}$, soit 12\%); l'augmentation semble certes minime sur le plan financier, mais est un véritable déclencheur sur le plan symbolique. À cette période de l'année, les sommes se négocient plus âprement dans les marchés, le ton monte très vite, et il n'est pas rare de voir marchands et clients en venir aux mains. D'ailleurs, cette année-là, alors que l'on était en plein mois saint, la ville avait été secouée par une histoire sordide où un chauffeur avait trouvé la mort: une foule hystérique avait forcé les portes de l'hôpital dans lequel elle l'avait précédemment envoyé, et l'avait défenestré1. Enfin, les étudiants de l'Université de Conakry, qui étaient en grève depuis près de deux mois, participaient de cette tension collective et la fin du mois de mars voyait de réels affrontements sur le campus, entre groupes rivaux ou entre ces groupes et les forces armées présentes dans l'enceinte de l'Université.

Il faut se garder naturellement d'affirmer que la rumeur a causé ces troubles: du moins les a-t-elle autant anticipés et reflétés ${ }^{2}$, que provoqués. La rumeur est donc bien moins "hors-temps"3 que 1'on pouvait le penser. Dans les trois exemples que nous venons de citer (au moins), la temporalité du surgissement est très nette: la rumeur se sert des tensions sociales comme d'un tremplin pour imposer ellemême son trouble. Si cette hypothèse était déjà admise dans le sens inverse (les situations de crise créent des rumeurs - Le Bon déjà en parlait! ${ }^{4}$ ), elle est plus difficile à imposer dans une acception non causale et non déterministe... La rumeur -ou plutôt son surgissementsemble donc enfin temporelle 5 .

1 J. DrAmou, "Déclaration du Ministre de l'intérieur: «Nul ne doit se faire justice»", Horoya, vol. 31, n 3644, 3 avril 1992, p. 12.

2 Nous plaidons pour une vision dynamique du social, dans laquelle la rumeur est à la fois précurseur et symptôme d'une réalité discrète.

3 A. GRYSPEERDT et A. KLEIN, op. cit., § 1.3 .

4 Gare à la confusion, car elle est fréquente: depuis Knapp jusqu’à Kapferer...

5 Il faut peut-être limiter la portée de la généralisation à ces rumeurs qui, dans le modèle reumaldien, sont celles bâties sur le modèle hystérique (sous cette bannière, nous rencontrons la rumeur d'Orléans bien sûr, mais aussi celle de l'anesthésistefantôme de Mattoon, l'épidémie des pare-brise de Seattle, les tracts anti-colorants de l'Hôpital de Villejuif, les tracts anti-décalcomanies au Isd, la maladie et la mort d'Isabelle Adjani): la contagion est "particulièrement rapide", 'le stade nymphal, 


\section{L'étude de la temporalité de la rumeur comme outill pour créer du sens?}

Après avoir illustré la difficulté du concept de rumeur à se figer dans le temps -et donc notre propre embarras à la définir explicitement-, nous nous sommes attaché à montrer le lien complexe qui liait rumeur et temps. Toute rumeur en effet peut être décomposée de triple façon: une thématique "hors du temps" car constamment actualisée -atemporelle (calquée sur le modèle de l'information journalistique, dont l'une des caractéristiques est justement d'être acontextuelle); un développement défiant le temps, éphémère et cyclique à la fois -bref, intemporel; et un surgissement dans le temps lié à des conditions sociales d'émergence-temporel. Reconstruire cette mosaïque de la temporalité (atemporalité, intemporalité et temporalité du phénomène) nous amène malgré nous à définir la rumeur, plus que nous l'avions espéré. La notion de temps nous permettrait-elle d'énoncer une définition où la rumeur est un phénomène éphémère, cyclique et conjoncturel de diffusion dans le socius d'un message à prétention d'actualité? Cela ferait de la rumeur non plus seulement un phénomène linguistique, mais un phénomène social dans lequel seraient mêlés à même degré d'importance le message oral et les signes sociaux manifestes.

Cette vision du phénomène permettrait d'aller plus loin encore dans l'étude de la temporalité du phénomène. La rumeur apparaît, on l'a vu, à l'occasion de tensions qui ne l'ont pas forcément engendrées. Mais pourrait-on lier alors la thématique aux conditions d'émergence à long terme? Une rumeur en effet ne peut toucher une population entière que parce qu'elle traite d'un problème commun à tous. L'étude de ce "problème commun" est facilitée par la conception d'une rumeur en tant que fait social: à condition d'abandonner temporairement la dichotomie discursif / extra-discursif, on peut utiliser l'étude de la réaction populaire, c'est-à-dire des signes manifestes de la rumeur pour utiliser des termes bysoviens, pour comprendre la thématique.

Dans le cas de la rumeur de Guinée, des faits troublants sur le surgissement de la crise peuvent nous guider. Si tous les auditeurs s'accordaient sur son côté vraisemblable (un guide touristique très 
populaire ${ }^{1}$ a même pour habitude de prévenir ses lecteurs"baroudeurs" en des termes crus tels que: «Souvent la femme africaine "boutique son cul"» ou «la jeune fille (...), après la nuit, attendra un "petit cadeau"»; cela reflète assez bien les relations entre les deux communautés, basées sur un déséquilibre économique qui perturbe le reste...), personne ne justifiait pour autant les actes des "voyous" dans la ville, les exactions "des bandes de casseurs". Et, fait étrange pour le sociologue, la populace ne s'en prenait qu' aux jeunes femmes habillées de manière non traditionnelle, et aux couples métissés: on assista à des déchaînements de violence contre ces deux groupes de victimes, les unes mises à nu, voire violées; les autres pourchassés et leurs grosses voitures $4 \times 4$ bombardées de pierre.

On pourrait poser l'hypothèse, en remarquant que la cible des émeutes étaient des éléments de la société dont la seule différence était le comportement vis-à-vis des coutumes et des traditions, que c'était également cela que la rumeur visait sans le dire. C'est en effet ce que l'on trouve dans la rumeur: un gardien surprend des conduites déviantes; il en saisit les autorités traditionnelles; le problème est résolu. La dialectique de l'ordre et du désordre, résumée en une histoire perverse... et voilà une capitale africaine en émoi, qu'est-ce à dire? Anodine en apparence, elle posait néanmoins sans vergogne les problèmes cruciaux avec lesquels le corps social se débattait: émancipation de la jeunesse, place des femmes dans la société, patriarcat, matérialisme... tout en utilisant des images dignes de celles d'Épinal. De là à lier la thématique de la rumeur avec la vie du corps social, il n'y a qu'un pas: le pays a connu en 1984 un revirement politique complet, passant de l'autarcie socialiste à un libéralisme quasi anarchique. De là naissent les soupirs nostalgiques de l'ordre ancien: "Dans l'Ancien régime, tu tenais ta langue et tu soignais ton comportement, sinon c'est le camp Alpha Yaya [camp militaire de sinistre mémoire]. Là, au moins, il n'y avait ni crime, ni vol, ni viol"; "Vous verrez la suite. Tout est permis maintenant. L'ouverture démocratique... oui, vers la débauche et le laisser-aller". La rumeur, dans son message et non plus seulement dans son surgissement, se donnerait-elle à interpréter? Dans ce cas, quelle méthodologie utiliser? Morin et al. avaient tenté une sorte de socio-psychanalyse. Mais quelle base de référence utiliser en d'autres lieux, en d'autres temps?

1 P. Cloaguen et al., Le Guide du routard. Afrique noire, Paris, Hachette, 1990, p. 45-46. 